\title{
Compass and Straightedge in the Poincaré Disk
}

\section{Chaim Goodman-Strauss}

The spirit of this article belongs to another age. Today, "geometry" is most often analytic; this is especially suited to the making of nice pictures by computer. But here we give a synthetic approach to the development of hyperbolic geometry; our constructions use only a Euclidean compass and Euclidean straightedge, and can be carried out by hand. Indeed, M.C. Escher used something like the methods we give here to produce his well known Circle Limit I, II, III, and IV prints [9].

In [6], H.S.M. Coxeter describes a remarkable correspondence with Escher. Having met at the 1954 International Congress of Mathematics in Amsterdam, Coxeter apparently sent Escher a paper in which a drawing of part of a tiling of the Poincaré disk appeared. Coxeter must have been quite pleased and surprised to find a print of Circle Limit I in his mail in December 1958. It is quite remarkable that the drawing in the paper Coxeter had sent Escher was not even as detailed as our Figure 1, and did not show the "scaffolding" Coxeter had used in its construction. Nonetheless Escher deduced and generalized the technique of its construction, producing incredibly fine tesselations of the Poincaré disk.

The technique for these constructions is quite ingenious and makes use of a certain duality between circular arcs and points inside the disk and points and Euclidean lines outside the disk, described at the end of Section 1. In [6], Coxeter only incidentally describes this duality; this is not quite enough to complete the construction and here we describe a full suite of available techniques.

I believe nothing in this article can possibly be original: surely this was all wellknown at the end of the nineteenth century just as it has long been forgotten at the dawn of the twenty-first. There are closely related figures in the works of Gauss, Beltrami, Poincaré, Schwarz, Klein, Fricke, Burnside, and others [16]. These sources are analytic or simply have other concerns; none, apparently, describe how these figures were produced.

"Scaffolding" such as that of Figure 1 was shown only rarely, but it did appear and indeed, it seems to have been rediscovered several times. It seems reasonable to assume that many, perhaps nearly all, of the illustrated tilings of the Poincare disk were drafted, synthetically, by techniques very similar to those presented here.

Remarkably, this may be the first detailed, explicit synthetic construction of triangle tilings of the Poincare disk to appear in the mathematical literature.

The setting is the Poincaré disk model of the hyperbolic plane. We need only a few properties of this model to carry out all of our synthetic constructions:

First, the points of the model are the points in the open unit disk in the Euclidean plane. Second, geodesics are circular arcs orthogonal to the unit circle. Inversion across such an arc is to be an isometry.

The locus of points equidistant from a given point $A$ is a Euclidean circle (though the Euclidean center of this circle is not $A$ !). This is consistent with our other properties: this locus must be invariant under inversion through any geodesic passing through $A$; circles orthogonal to every geodesic through $A$ are the only curves satisfying this requirement.

Finally, angles in the Poincaré model are Euclidean angles. This is the only consistent choice, as inversion preserves Euclidean angles. 


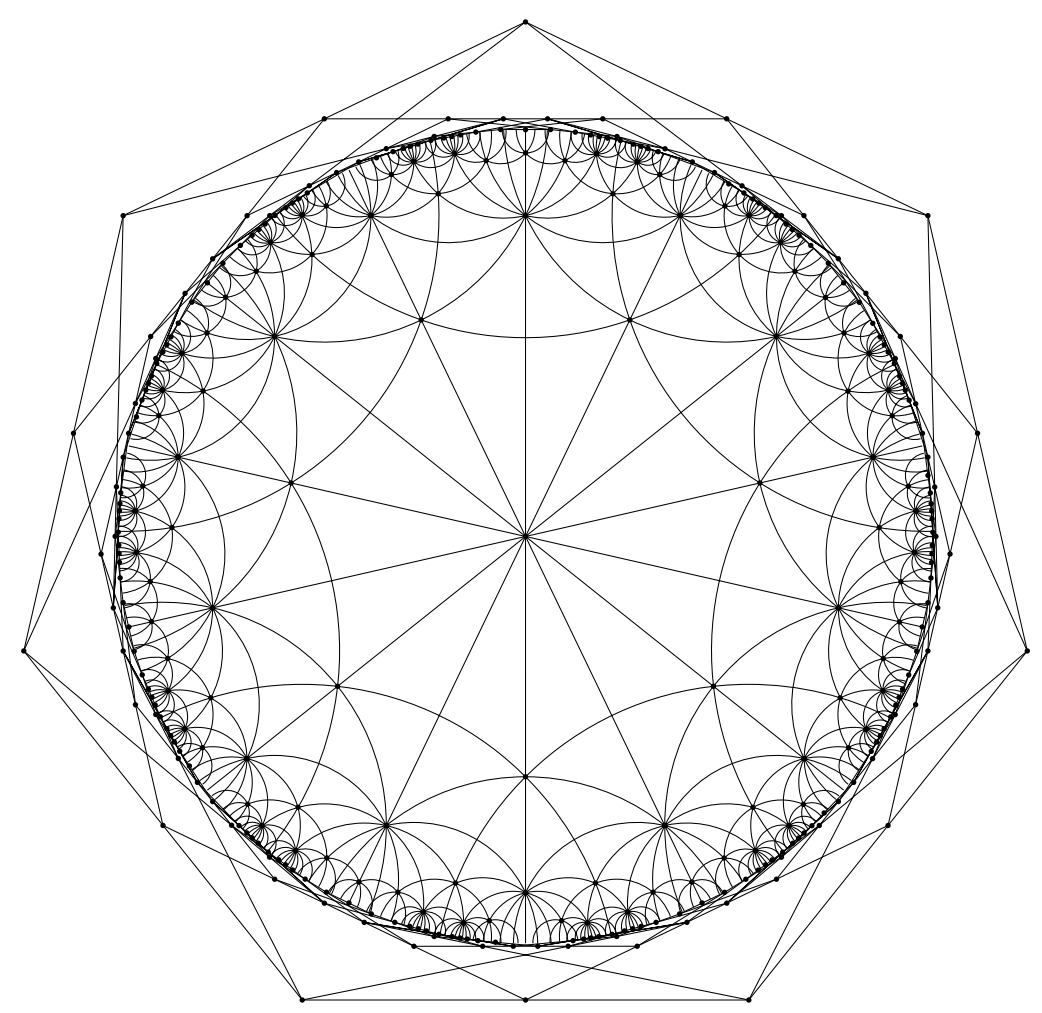

Figure 1. Synthetically constructed triangle tiling in $\mathbb{H}^{2}$.

Though this is enough to completely specify the metric (up to some normalization), we simply note that there is a metric satisfying these properties. There are, of course, dozens of excellent references; several distinctly interesting accounts appear in $[\mathbf{1}]-[\mathbf{5}],[7],[\mathbf{1 2}]-[\mathbf{1 5}],[\mathbf{1 7}]$, and [18]. A recent, related discussion, though analytic in treatment, appears in [8].

Some readers raised on an analytic treatment, with its abundance of hyperbolic trigonometric functions, natural logarithms and the like, may find the synthetic approach, using only Euclidean circles and lines, somewhat surprising.

But all facts about hyperbolic lines and circles are merely facts about Euclidean lines and circles (not respectively), via the Poincaré model. This is, of course, how Beltrami first showed that hyperbolic geometry was no less consistent than Euclidean geometry (though he used a different model).

For example, in the Poincaré disk, the existence of a unique hyperbolic geodesic between two given points is simply the existence of a unique circle, passing through the two given points, that is orthogonal to some given circle. It may not be obvious that such a circle exists - we give the construction shortly — but the point is that we can use synthetic techniques quite readily. And though we work only in the Poincare disk, it is straightforward to find similar constructions in the Klein disk and the upper half plane models.

In the first section, we give some basic useful constructions in the Euclidean plane. In the next section we show how to construct a hyperbolic straightedge and compass. Once these are in hand, we give several valuable constructions in the hyperbolic plane. In the final section we give techniques for constructing various tesselations of the hyperbolic plane with compass and straightedge. 
Geometer's Sketchpad sketches and scripts to accompany this article can be found on the author's website, http://comp.uark.edu/ cgstraus; David Flesner has prepared similar sketches, optimized for the Klein model [10].

1. USEFUL ELEMENTARY CONSTRUCTIONS. We take as given that the reader knows how to bisect a line segment, draw a parallel through a given point, draw a perpendicular through a given point, and perform other elementary Euclidean constructions.

We need a few other slightly less elementary constructions, which we give for completeness.

Construction 1.1. Construct a circle through three given non-collinear points $\mathrm{A}, \mathrm{B}, \mathrm{C}$.

Construct segments $\overline{\mathrm{AB}}, \overline{\mathrm{BC}}$. Construct the perpendicular bisectors $\ell_{1}, \ell_{2}$ of these segments. Let $O$ be the point of intersection of $\ell_{1}, \ell_{2}$; the desired circle has center $O$ and passes through, say $A$.

Construction 1.2. Invert a point through a circle $\mathcal{C}$ with center $\mathrm{O}$.

There are a many methods; here is one:

If our point $B$ is outside the circle, construct segment $\overline{\mathrm{OB}}$ as at the left in Figure 2. Construct the circle $\mathcal{C}^{\prime}$ passing through $\mathrm{O}$, with center at the midpoint of this segment. Let $\mathrm{P}$ be a point of intersection of $\mathcal{C}$ and $\mathcal{C}^{\prime}$ (note that $\triangle \mathrm{OPB}$ is a right triangle). Now take the perpendicular $\ell$ to $\overline{\mathrm{OB}}$ through $\mathrm{P}$. Let $\mathrm{A}$ be the point of intersection of $\ell$ with $\overline{\mathrm{OB}}$. Then $\mathrm{A}$ is the inverse of $\mathrm{B}$ with respect to $\mathcal{C}$.

If we wish to invert a point $A$ lying inside the circle (other than $O$ !) simply reverse this process. Take the perpendicular $\ell$ through $A$ to $\overline{\mathrm{OA}}$. Let $\mathrm{P}$ be the point of intersection of $\ell$ and $\mathcal{C}$. Let $\ell^{\prime}$ be the perpendicular to $\overline{\mathrm{OP}}$ passing through $\mathrm{P}$. Then the point $\mathrm{B}$ of intersection of $\overleftrightarrow{\mathrm{OA}}$ and $\ell^{\prime}$ is the desired inverse of $\mathrm{A}$ through $\mathcal{C}$.

There is a significantly quicker method if a little additional structure is given:

Construction 1.3. Given a pair of orthogonal circles $\mathcal{C}, \mathcal{C}^{\prime}$ with centers $\mathrm{O}, \mathrm{O}^{\prime}$, respectively, and a point $\mathrm{A}$ on $\mathcal{C}^{\prime}$, construct the inverse of $\mathrm{A}$ with respect to $\mathcal{C}$.

Simply construct the ray $\overrightarrow{O A}$ as at the right in Figure 2; one of the two points of intersection of $\overrightarrow{O A}$ with $\mathcal{C}^{\prime}$ is $A$; the other, $B$, is the inverse of $A$ with respect to $\mathcal{C}$.
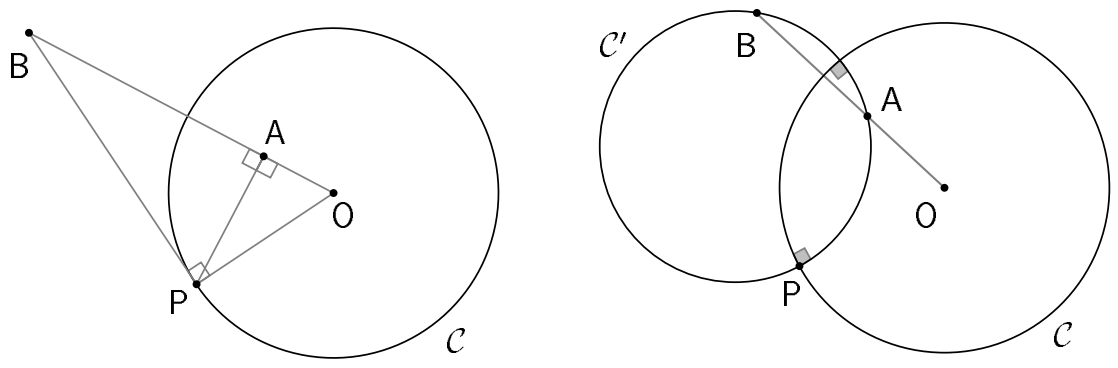

Figure 2. Constructions 1.2 and 1.3. 
One proves that this gives the desired point by considering the similar triangles $\triangle \mathrm{OAP}$ and $\triangle \mathrm{OPB}$, where $\mathrm{P}$ is a point of intersection of $\mathcal{C}$ and $\mathcal{C}^{\prime}$. An immediate consequence is:

Lemma 1.4. Let $\mathcal{C}, \mathcal{C}^{\prime}$ be orthogonal circles and let $\mathrm{A}$ be a point on $\mathcal{C}^{\prime}$. Then the inverse of $\mathrm{A}$ with respect to $\mathcal{C}$ also lies on $\mathcal{C}^{\prime}$. Similarly, if $\mathrm{A}$ and $\mathrm{B}$ are inverses with respect to $\mathcal{C}$, lying on a circle $\mathcal{C}^{\prime}$, then $\mathcal{C}$ and $\mathcal{C}^{\prime}$ are orthogonal.

Consequently:

Lemma 1.5. Let $\mathcal{C}$ be a circle with center $\mathrm{O}$. Let $\mathrm{A}$ be a point other than $\mathrm{O}$. Then the locus of centers of all circles passing through $\mathrm{A}$ and orthogonal to $\mathcal{C}$ is a straight line. If $\mathrm{A}$ is in the interior of [on, in the exterior of] $\mathcal{C}$ this line is in the exterior of [tangent to, in the exterior of] $\mathcal{C}$. Finally, any line in the exterior of, or tangent to, $\mathcal{C}$ is such a locus.

Proof. Any circle through $\mathrm{A}$ and orthogonal to $\mathcal{C}$ must pass through the inverse $\mathrm{B}$ of $A$ with respect to $\mathcal{C}$. Consequently, if $A, B$ are distinct (i.e., not on $\mathcal{C}$ ), the center of this circle must lie on the perpendicular bisector of $\overline{\mathrm{AB}}$, which is in the exterior of $\mathcal{C}$. Conversely, any circle with center on this bisector passes through both $A$ and $B$ and so is orthogonal to $\mathcal{C}$.

If $\mathrm{A}$ lies on $\mathcal{C}$, then the locus is clearly the line tangent to $\mathcal{C}$ at $\mathrm{A}$.

It is trivial to show that any line in the exterior of, or tangent to $\mathcal{C}$ is such a locus.

We further note:

Construction 1.6. Given a circle $\mathcal{C}$ with center $\mathrm{O}$, and point $\mathrm{A}$ in the exterior of $\mathcal{C}$, construct the unique circle $\mathcal{C}^{\prime}$ with center $\mathrm{A}$, orthogonal to $\mathcal{C}$.

Let $\mathcal{C}^{\prime \prime}$ be the circle with center at the midpoint of segment $\overline{\mathrm{AO}}$ and passing through $A$ and $O$, as at the left in Figure 3; let $P$ be a point in the intersection of $\mathcal{C}$ and $\mathcal{C}^{\prime}$. Then the desired circle has center $A$ and passes through $P$.

Lemma 1.5 and Construction 1.6 establish a very important fact: with respect to a given fixed circle $\mathcal{C}$, orthogonal circles $\mathcal{C}^{\prime}$ are in correspondence with points in the exterior of $\mathcal{C}$ (the centers of the $\mathcal{C}^{\prime}$, called their poles) and points in the interior of $\mathcal{C}$ correspond to straight lines (called their polars) in the exterior of $\mathcal{C}$.
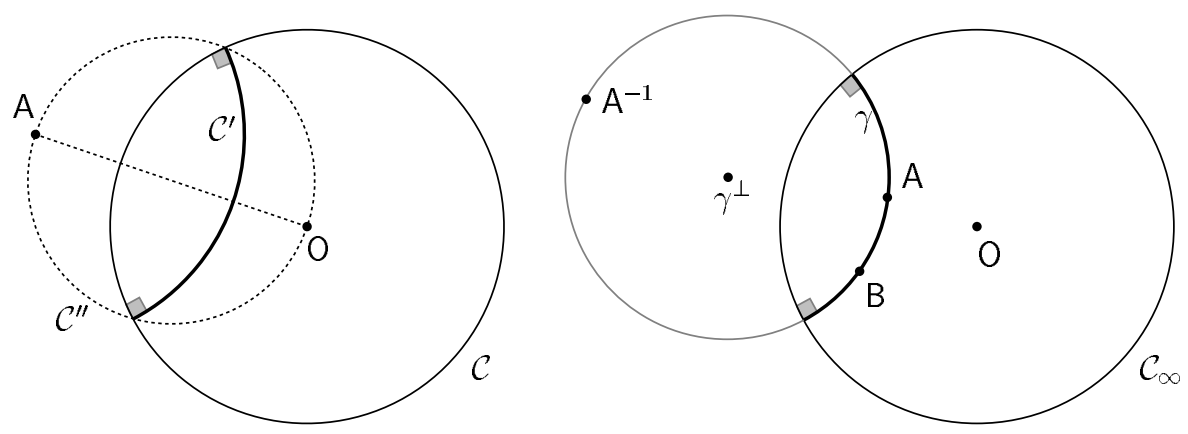

Figure 3. Constructions 1.6 and 2.1. 


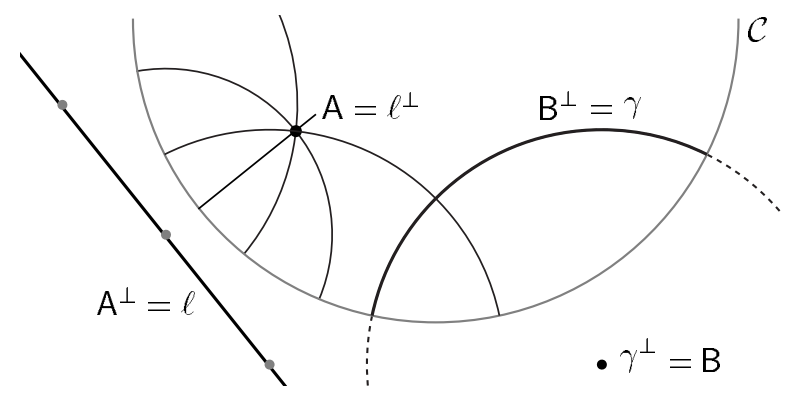

Figure 4. Poles and polars.

Assuming, then, that a specific circle $\mathcal{C}$ has been fixed, denote the pole of a circle $\gamma$ by $\gamma^{\perp}$; conversely, each point $B$ in the exterior of $\mathcal{C}$ is the pole of some circle, denoted by $B^{\perp}$, as illustrated in Figure 4 . Similarly, denote the polar of each point $A$ in the interior of $\mathcal{C}$ by $\mathrm{A}^{\perp}$; conversely, each line $\ell$ not meeting $\mathcal{C}$ is the polar of some point, denoted by $\ell^{\perp}$.

Properly speaking, the poles of our geodesics reside in the real projective plane $\mathbb{R} P^{2}$ : we must adjoin points at infinity to account for the poles of geodesics that are diameters of $\mathcal{C}$. This gives a simple proof that the space of lines in $\mathbb{H}^{2}$ is an open Mobius band, i.e., $\mathbb{R} P^{2}$ less the closed disk bounded by $\mathcal{C}$.

\section{CONSTRUCTING THE HYPERBOLIC STRAIGHTEDGE AND COM-}

PASS. Now we construct the hyperbolic straightedge and compass. We always take as given $\mathcal{C}_{\infty}$, the circle at infinity in the Poincaré disk model of the hyperbolic plane, and $\mathrm{O}$, the center of this circle. The open disk itself is denoted by $D$. When we refer to inverses, unless otherwise noted, we mean inverses with respect to $\mathcal{C}_{\infty} ; X^{\perp}$ means the polar, pole, etc. of some object $X$ with respect to $\mathcal{C}_{\infty}$, in the sense described at the end of Section 1.

Construction 2.1. Given points $\mathrm{A}, \mathrm{B} \in D$, construct the hyperbolic geodesic $\stackrel{\mathfrak{A B}}{\mathrm{B}}$. Equivalently, given two points $\mathrm{A}, \mathrm{B}$ and a circle $\mathcal{C}_{\infty}$ with center $\mathrm{O}$, construct the unique circle through $\mathrm{A}, \mathrm{B}$ that is orthogonal to $\mathcal{C}_{\infty}$.

This is wonderfully simple: As at the right in Figure 3 , invert $A$ through $\mathcal{C}_{\infty}$ to construct $A^{-1}$ and the desired circle is the circle $\gamma$ through $A, B$, and $A^{-1}$. Lemma 1.4 ensures that the constructed circle is orthogonal to $\mathcal{C}_{\infty}$.

Hyperbolic geodesics are then in precise correspondence with points outside the Poincare disk, and points within the Poincaré disk are in precise correspondence with (Euclidean) lines in the exterior of the disk. This gives us a very rapid method of constructing the geodesic between two points $\mathrm{A}, \mathrm{B}$ if the polars $\mathrm{A}^{\perp}$ and $\mathrm{B}^{\perp}$ happen to be provided:

Construction 2.2. Given points $\mathrm{A}, \mathrm{B} \in D$ and their polars $\mathrm{A}^{\perp}$ and $\mathrm{B}^{\perp}$, construct $\stackrel{\mathrm{AB}}{\stackrel{1}{1}}$

As illustrated at the left in Figure 10, letting $P$ be the point of intersection of $A^{\perp}$ and $\mathrm{B}^{\perp}$, the desired geodesic is simply $\mathrm{P}^{\perp}$, the circle with center $\mathrm{P}$ passing through A, B.

In practice, if $\mathrm{A}$ and $\mathrm{B}$ are relatively close to each other in $D, \mathrm{~A}^{\perp}$ and $\mathrm{B}^{\perp}$ are nearly parallel and $\mathrm{P}$ is hard to locate precisely. Fortunately, the perpendicular bisector of 


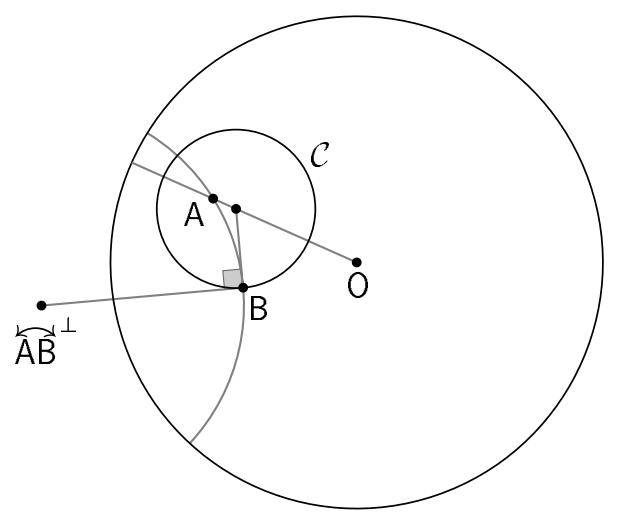

Figure 5. Construction 2.3.

the (Euclidean) segment $\overline{\mathrm{AB}}$ must also pass through $\mathrm{P}$, increasing the accuracy of the construction tremendously.

We next construct the hyperbolic compass.

Construction 2.3. Given points $\mathrm{A}$ and $\mathrm{B}$, construct the hyperbolic circle with hyperbolic center $\mathrm{A}$, passing through $\mathrm{B}$.

We take as given that the desired curve $\mathcal{C}$ is the unique Euclidean circle that is orthogonal to every geodesic through $A$. In particular, then, $\mathcal{C}$ must be orthogonal to the Euclidean line $\overleftrightarrow{\mathrm{OA}}$ and hence its center is on this line. Moreover, $\mathcal{C}$ must be orthogonal to $\overparen{A B}$; thus the center of $\mathcal{C}$ must lie on the tangent to $\overparen{A B}$ at $B$. Hence, as in Figure 5, we take $\mathcal{C}$ to be the Euclidean circle with center at the intersection of these two lines, and passing through $B$.

\section{SEVERAL ELEMENTARY CONSTRUCTIONS IN THE HYPERBOLIC}

PLANE. A large number of useful constructions make no assumption of the parallel postulate and so work in the hyperbolic plane, substituting our hyperbolic straightedge and compass for the Euclidean compass and straightedge.

Construction 3.1. Given $\mathrm{A}, \mathrm{B} \in D$, construct the perpendicular bisector of the segment $\overparen{\mathrm{AB}}$. Similarly, construct the midpoint of the segment $\overparen{\mathrm{AB}}$.

The construction is precisely the same as in the Euclidean plane, but with our hyperbolic compass and straight edge. As at left in Figure 6, draw the two hyperbolic circles $\mathcal{C}_{1}, \mathcal{C}_{2}$ centered at $A, B$ and passing through $B, A$. Let $P, Q$ be the points of $\mathcal{C}_{1} \cap \mathcal{C}_{2}$. Then $\stackrel{\mathrm{PQ}}{ }$ is the desired perpendicular bisector and the midpoint of $\overparen{\mathrm{AB}}$ is the intersection of $\stackrel{2}{\mathrm{PQ}}$ and $\stackrel{\mathrm{AB}}{\text {. }}$.

In fact, though, this construction can be carried out much more efficiently:

(Alternate version) Inversion across the perpendicular bisector $\gamma$ of $\overparen{A B}$ interchanges $A$ and $B$; moreover since $\gamma$ is to be orthogonal to $\mathcal{C}_{\infty}$, this inversion must also interchange $A^{-1}$ and $B^{-1}$. Hence, $\gamma^{\perp}$ lies on both $\overleftrightarrow{A B}$ and $\overleftrightarrow{A^{-1} B^{-1}}$. At the right in 


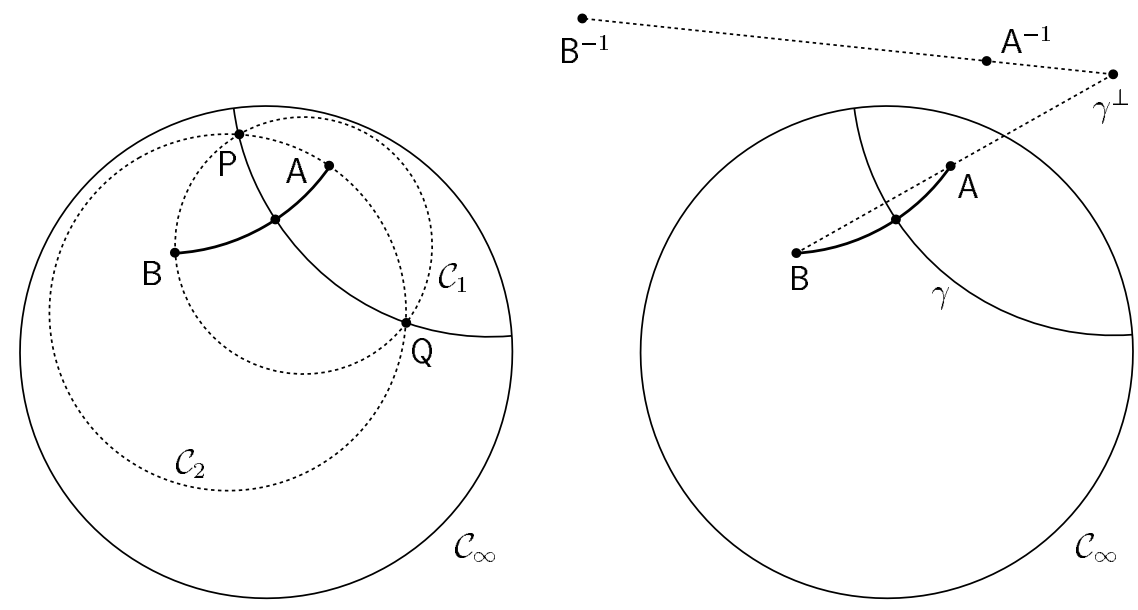

Figure 6. Construction 3.1: On the left, a modification of the usual Euclidean construction. On the right, a more efficient version.

Figure 6, we take the point of intersection as $\gamma^{\perp}$, and consequently Construction 1.6 gives us the desired bisector $\gamma$.

Similarly, we can easily mark off equal divisions in a line, etc. by adapting the Euclidean constructions, though simpler, more direct techniques may be available. Here are a few more useful constructions:

Construction 3.2. Given a point $\mathrm{A} \in D$ and a geodesic $\gamma$, construct the hyperbolic reflection of A across $\gamma$. Similarly, "translate" a given hyperbolic triangle $\triangle \mathrm{ABC}$ to a given location (specified by the images $\mathrm{A}^{\prime}, \mathrm{B}^{\prime}$ of $\mathrm{A}, \mathrm{B}$ ).

First, to reflect a point $\mathrm{A}$ across $\gamma$, simply invert $\mathrm{A}$ through $\gamma$, using Construction 1.3. For the second part, we need to construct the image of $C$; there are two possibilities, depending on whether we wish the transformation to be orientation preserving or reversing.

We follow exactly the same construction as in the Euclidean case. We first construct the perpendicular bisector $\gamma_{1}$ of the segment ${\widehat{A A^{\prime}}}^{\prime}$. Then let $B^{\prime \prime}$ be the inverse of $B$ across $\gamma_{1}$. Then let $\gamma_{2}$ be the geodesic through $A^{\prime}$ and the midpoint of $\mathrm{B}^{\prime} \mathrm{B}^{\prime \prime}$. Hence inverting first through $\gamma_{1}$ and then through $\gamma_{2}$ takes $A, B$ to $A^{\prime}, B^{\prime}$. Let $C^{\prime}$ be the image of $C$ under this operation. If we wish our transformation to be orientation preserving we are done. Otherwise, invert once more across the geodesic $\mathrm{A}^{\prime} \mathrm{B}^{\prime}$.

We next adapt the standard Euclidean proof to show that the "collapsible" hyperbolic compass of Construction 2.3 is equivalent to a "rigid" hyperbolic compass.

Construction 3.3. Given points $\mathrm{A}, \mathrm{B}, \mathrm{C} \in D$, construct the hyperbolic circle with hyperbolic center $\mathrm{A}$ and with radius congruent to the hyperbolic segment $\overparen{\mathrm{BC}}$.

First construct the perpendicular bisector $\gamma$ of the segment $\overparen{B A}$. Then invert $C$ across $\gamma$ to obtain $P$. The desired circle has hyperbolic center $A$ and passes through $P$.

We conclude this section with a useful and simple construction: 
Construction 3.4. Given a geodesic $\gamma$, a point $\mathrm{A} \in \gamma$, and a Euclidean angle $\alpha$, construct a geodesic meeting $\gamma$ at $\mathrm{A}$ with angle $\alpha$.

As in Figure 9 let $\ell$ be the line passing through $\gamma^{\perp}$ and $A$ and let $\ell^{\prime}$ be the result of rotating $\ell$ by $\alpha$ about $A$. Then the desired geodesic is $\mathrm{P}^{\perp}$, where $\mathrm{P}$ is the intersection of $\ell^{\prime}$ and $\mathrm{A}^{\perp}$.

4. TRIANGLE TILINGS OF THE HYPERBOLIC PLANE. We now turn to the construction of "triangle tilings" of the hyperbolic plane; there are many descriptions of such tilings and their connections. A nice early treatment (complete with synthetically drafted illustrations!) appears in [11]. Keeping things elementary and synthetic, we say that such a tiling is described, up to hyperbolic isometries, by an unordered triple of whole numbers $p, q, r$ with $p^{-1}+q^{-1}+r^{-1}<1$. One can prove by a variety of methods that there is a tiling of the hyperbolic plane by triangles with interior angles $\pi / p, \pi / q, \pi / r$, generated by inversions across the sides of some base triangle.

We now give an explicit construction of such tilings, first for the triple 7, 4, 2 and then for all triples of the form $p, q, 2$. The reader is invited to generalize.

Each case consists of two tasks: First, construct a triangle with the appropriate angles and then, second, extend this into a tiling. For the first task, we assume that the appropriate Euclidean angles have been provided (thus we are not asking that these angles are themselves constructible!). The second task can be carried out trivially, but quite tediously, by repeated applications of Construction 3.2. However, we give an elaboration of an elegant technique implicit in [6]. In this paper, Coxeter describes correspondence with Escher leading to the construction of Escher's famous Circle Limits I-IV. The method described in that paper, in effect, repeated applications of Construction 2.2, is not quite sufficient to carry on indefinitely, though, but we have provided additional tricks. Escher must have discovered these or very similar devices on his own.

4.1. Constructing the $7,4,2$ Triangle Tiling. Our first task is to construct a hyperbolic triangle with angles $\pi / 7, \pi / 4, \pi / 2$. Of course, an angle of $\pi / 7$ is not constructible, but such an angle certainly exists! We take this angle as given. It is convenient to construct the circle $\mathcal{C}_{\infty}$ as an artifact of the construction of the triangle (rather than beginning with $\mathcal{C}_{\infty}$ ).

Step 1: (Figure 7) Choose two points $\mathrm{O}$ and $\mathrm{A}$. Let $\rho$ denote the (Euclidean) rotation about $\mathrm{O}$ by $2 \pi / 7$. Our first geodesic $\gamma$ is the (Euclidean) circle centered at the midpoint $\mathrm{B}$ of $\overline{\mathrm{A} \rho(\mathrm{A})}$ and passing through $\mathrm{A}$ and $\rho(\mathrm{A})$. Now we need $\mathcal{C}_{\infty}$ : let $\mathcal{C}$ be the circle centered at the midpoint of $\overleftrightarrow{\mathrm{BO}}$ and let $\mathrm{C}$ be a point of $\mathcal{C} \cap \gamma$. Then let $\mathcal{C}_{\infty}$ be the circle with center $\mathrm{O}$ passing through $\mathrm{C}$. We now note that:

(i) $\mathcal{C}_{\infty}$ and $\gamma$ are orthogonal, (ii) $\gamma$ is orthogonal to the line $\overleftrightarrow{\mathrm{BO}}$, and (iii) $\gamma$ meets the line $\overleftrightarrow{A O}$ at an angle of $\pi / 7$ (this can be seen by inspecting the angle between the lines $\overleftrightarrow{A O}$ and $\overleftrightarrow{A B}$ ).

Consequently, letting $\mathrm{P}, \mathrm{Q}$ be the points of intersection of $\gamma$ with $\overleftrightarrow{\mathrm{AO}}, \overleftrightarrow{\mathrm{BO}}$ in the interior of $D$, the hyperbolic triangle $\triangle \mathrm{OPQ}$ has interior angles $\pi / 7, \pi / 7, \pi / 2$.

The geodesic $A^{\perp}$ must pass through $Q$ (since the line $\overleftrightarrow{A B}$ must be the polar of $Q$ ); it is easy to show that $A^{\perp}$ meets $\overleftrightarrow{O B}$ at an angle of $\pi / 4$ ( $\triangle A B P$ is a Euclidean isoceles right triangle by its construction from $\gamma$ ). Moreover, $A^{\perp}$ must be orthogonal to $\overleftrightarrow{A O}$. Hence, letting $R$ be the point of intersection of $A^{\perp}$ and $\overleftrightarrow{A O}$ in the interior of $D, \triangle O R P$ and $\triangle P Q R$ have interior angles $\pi / 7, \pi / 4, \pi / 2$. 

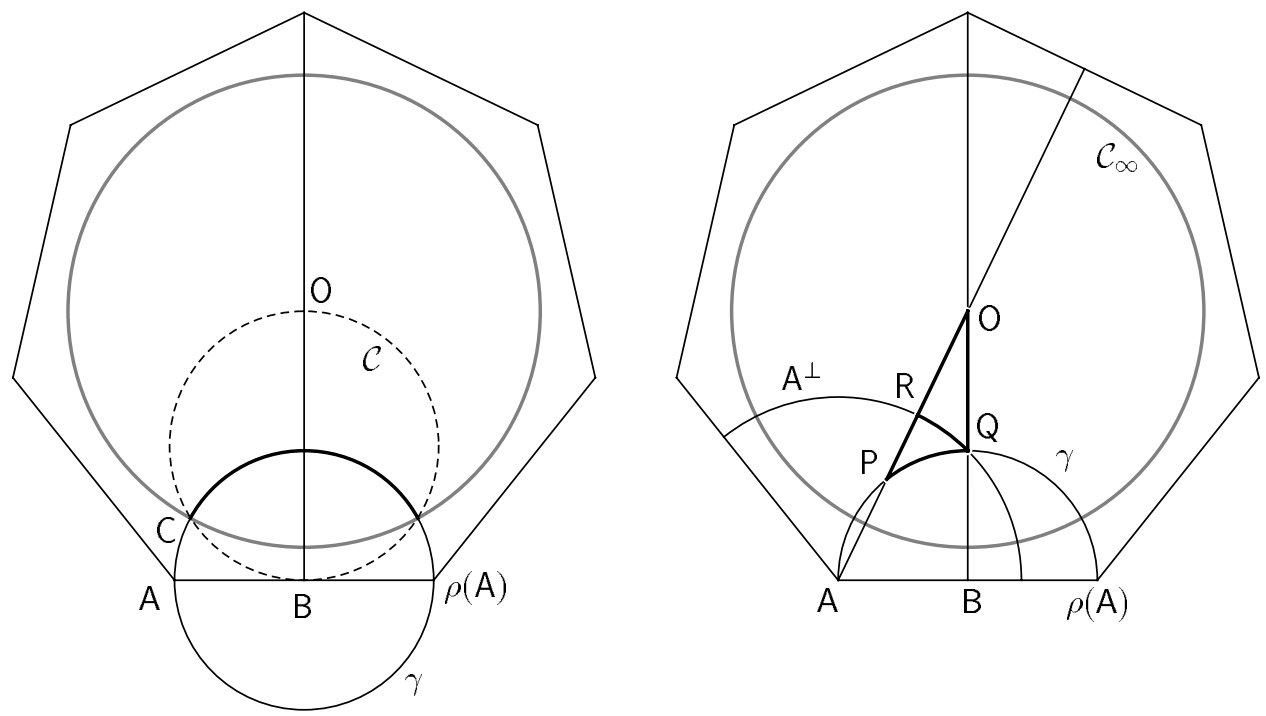

Figure 7. Constructing a 7, 4, 2 triangle.

Exactly this same procedure can be used to produce hyperbolic triangles with internal angles $\pi / p, \pi / 4, \pi / 2$ for any $p \geq 5$.

Step 2: We now need to tile the Poincaré disk by congruent images of our triangle.

The crudest, but most general (and clearly successful) method is to invert the points

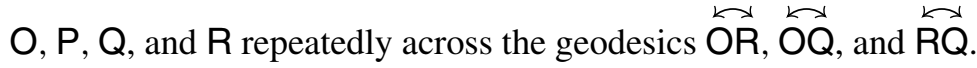

However, we can do much better. At each stage, we automatically take advantage of the dihedral symmetry in our tiling; so we assume that once any point, geodesic, pole, or polar is created, its images under this symmetry are also created. So, we are now faced with Figure 8.

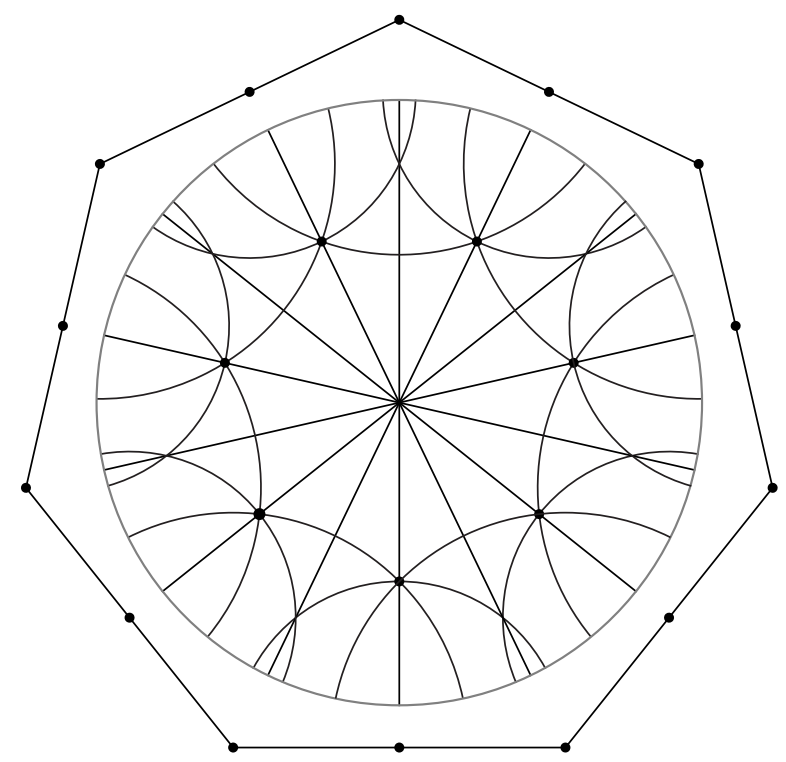

Figure 8. Beginning the 7, 4, 2 triangle tiling. 

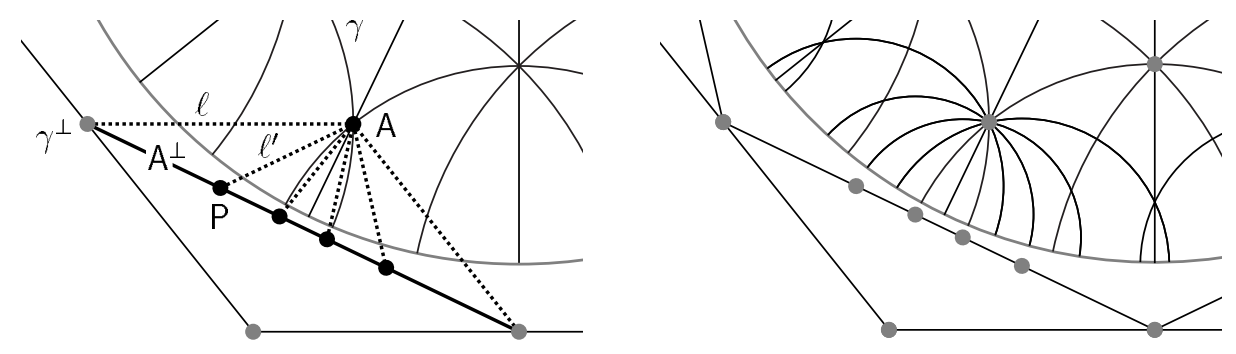

Figure 9. Rotating geodesics about a point.
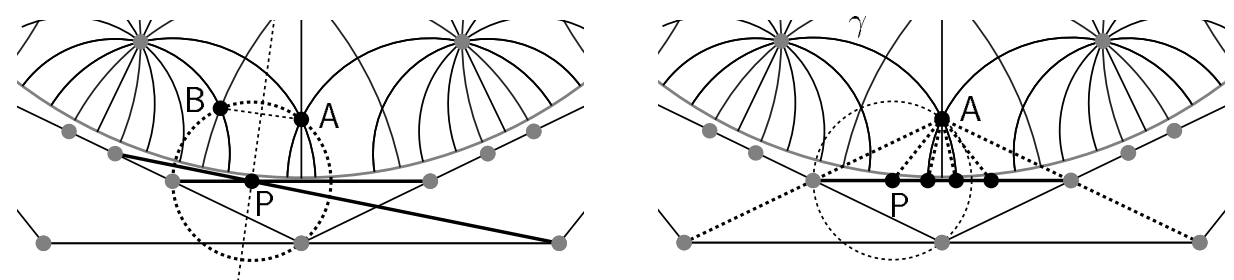

Figure 10. Efficiently producing a geodesic through two points and rotating a geodesic about a point.

Our next task is to add geodesics about the point $A$ in Figure 9. This is effectively the same as rotating the geodesic $\gamma$ by $\pi k / 7$ about $\mathrm{A}$ : apply Construction 3.4 as illustrated in Figure 9.

Similarly, we next need to add a geodesic between points A, B at the left in Figure 10. But this is just an application of our Construction 2.2. Note how much quicker this is than Construction 2.1! Alternatively, as at the right in Figure 10 we might go ahead and rotate the geodesic $\gamma$ by $\pi k / 4$, which gives us not only the geodesic $\overparen{A B}$ but a few others besides.

It should be clear that we can apply these two techniques repeatedly, using inversion if we ever get stuck, fleshing out both the internal and external structures, until we have generated as much of the triangle tiling as we desire. We have carried this process out a bit further in Figure 1

4.2. Constructing the $\boldsymbol{p}, \boldsymbol{q}, \mathbf{2}$ Triangle Tiling. We describe only the first step, the construction of a hyperbolic triangle with internal angles $\pi / p, \pi / q, \pi / 2, p^{-1}+q^{-1}<$ $1 / 2$.

But the technique is pretty straightforward. In Figure 11 we illustrate the procedure for $p=5, q=8$. Begin as before with two points $\mathrm{O}$, $\mathrm{A}$; rotate $\mathrm{A}$ about $\mathrm{O}$ by $\pi / p$ to produce $\mathrm{A}^{\prime}$. Let $\mathrm{P}$ be the midpoint of $\overrightarrow{\mathrm{AA}^{\prime}}$. Now rotate $\overleftrightarrow{\mathrm{AA}^{\prime}}$ by $\pi / q-\pi / p$ around $\mathrm{A}$ to produce $\ell$ (our rotations are by signed angles), and let $B$ be the intersection of $\ell$ with $\overleftrightarrow{\mathrm{OP}}$. Our first geodesic is $\gamma$, the circle centered at $B$, passing through $A, A^{\prime}$.

Let $\mathcal{C}$ be the circle passing through $\mathrm{O}$ and $\mathrm{B}$, centered at the midpoint of $\overline{\mathrm{OB}}$, and let $\mathrm{C}$ be a point of intersection of $\gamma$ with $\mathcal{C}$. Then $\mathcal{C}_{\infty}$ is to be the circle centered at $\mathrm{O}$ passing through $\mathrm{C}$.

Finally, let $R$ be the intersection of $\gamma$ with $\overleftrightarrow{O A}$ and let $Q$ be the intersection of $\gamma$ with $\overleftrightarrow{\mathrm{OB}}$. It is not hard to show that the hyperbolic triangle $\triangle \mathrm{OQR}$ has internal angles $\pi / p, \pi / q, \pi / 2$.

Once we have this triangle, we proceed exactly as before to produce a tiling. 

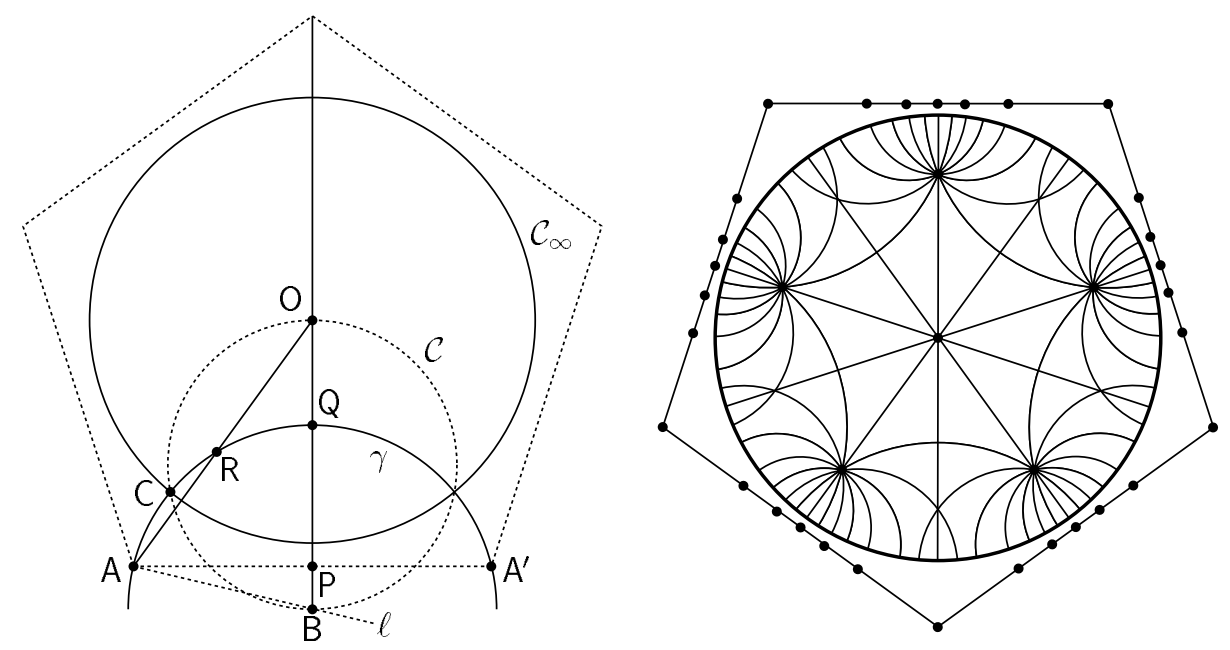

Figure 11. Constructing a $p, q, 2$ triangle.

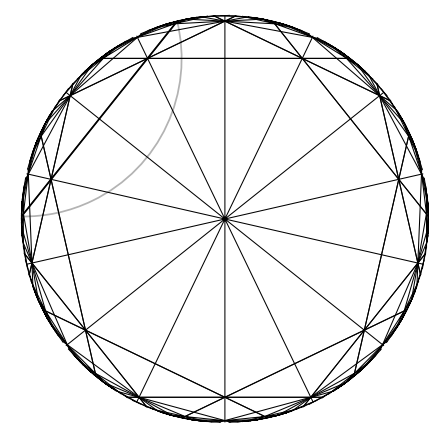

Figure 12. The 742 triangle tiling in the Klein model.

4.3. Variations. It is not hard to find a method for producing a $p, q, r$ triangle for any triple $p, q, r$ with $p^{-1}+q^{-1}+r^{-1}<1$.

We can create tilings that lack the dihedral symmetry of Figure 1 by applying Construction 3.2 to reflect our base triangle across some arbitrary geodesic before generating the tiling.

We can create tilings with more intricate shapes, based on the symmetry of the triangle tiling. Of course, this is exactly what Escher did in his Circle Limit prints.

We can easily convert our constructions to the Klein model: a geodesic in the Klein model has the same intersection with $\mathcal{C}_{\infty}$ as the corresponding geodesic in the Poincare disk. Hence, we can produce poles, polars, points, geodesics, angles, and so forth readily by converting back to the Poincaré model. In Figure 12 we illustrate the tiling of Figure 1 in the Klein model. A single arc has been highlighted and illustrated in both models.

There is no end of possibilities. It is wonderfully satisfying to make these pictures by hand, patiently, with pencil and paper, compass and straightedge. I encourage you to test this theorem for yourself!

ACKNOWLEDGMENTS. I am grateful to John Stillwell, H.S.M. Coxeter, Doris Schattschneider, David Flesner, Lee Dickey, Doug Dunham, and Donald Crowe for their help putting this paper in historical perspective. 
1. M. Berger, Geometrie, vols. 1 and 2, Springer-Verlag, New York, 1987.

2. R. Bonola, Non-Euclidean geometry, Dover, New York, 1955.

3. W. Burnside, Theory of groups of finite order, Cambridge University Press, Cambridge, 1911.

4. J.W. Cannon, W.J. Floyd, R. Kenyon, and W.R. Parry, Hyperbolic Geometry, Flavors of Geometry, S. Levy, ed., MSRI Publications 31 (1997) 59-115.

5. H.S.M. Coxeter, Non-Euclidean geometry, University Toronto Press, Toronto, 1942.

6. H.S.M. Coxeter, The non-Euclidean symmetry of Escher's picture "Circle Limit III", Leonardo 12 (1979) $19-25$.

7. H.S.M. Coxeter, The trigonometry of Escher's woodcut "Circle Limit III", Math. Intelligencer 18:4 (1996) 42-46.

8. H.S.M. Coxeter, The trigonometry of hyperbolic tesselations, Canad. Math. Bull. 40 (1997) 158-168.

9. M.C. Escher, The world of M.C. Escher, H.N. Abrams, New York, 1971.

10. D. Flesner, private communication.

11. F. Klein, Über die Transformation siebenter Ordnung der elliptischen Funktionen, Math. Ann. 14 (1879) 428-471.

12. M. Kline, Mathematical thought from ancient to modern times, vols. 1-3, Oxford University Press, New York, 1972.

13. E.G. Rees, Notes on Geometry, Springer-Verlag, New York, 1983.

14. B.A.W. Russell, An Essay on the Foundations of Geometry, Cambridge University Press, Cambridge, 1897.

15. J. Stillwell, Geometry of surfaces, Springer-Verlag, New York, 1992.

16. J. Stillwell, Sources of hyperbolic geometry, American Mathematical Society, Providence, R.I., 1996.

17. W.P. Thurston, Three-dimensional geometry and topology, Princeton University Press, Princeton, 1997.

18. I. Tóth, Non-Euclidean geometry before Euclid, Scientific American 221:5 (Nov., 1969) 87-98.

C. GOODMAN-STRAUSS received his Ph.D. at the University of Texas in Austin in 1994, in knot theory, under the supervision of John Luecke. He has been at the University of Arkansas since, with some time spent at the Geometry Center at the University of Minnesota. His current research focus is the study of aperiodic tiles and the complexity of tilings. He is the founder of the world's foremost fictional trading card company, Ptolemy Mathcard, and has recently taken up chainsaw sculpture.

University of Arkansas, Fayetteville AR 72701

cgstraus@comp.uark.edu 